\section{Haemoglobinometry by an automatic analytical procedure}

\author{
M. G. NELSON AND A. LAMONT From the Royal \\ Victoria Hospital, Belfast
}

In any busy laboratory providing a haematological service, the determination of haemoglobins by a manual method consumes much technical time. In our laboratory, out of a total of 79,000 routine haematological tests performed last year, $35 \%$ were haemoglobin estimations.

As part of a programme of automation of general haematology, we have, for six months, carried out the haemoglobin determinations with an Autoanalyser (Technicon Instruments Ltd.). This has proved easy, accurate, and time-saving. Our experience with this machine for this purpose has so impressed us that we are prompted to bring the procedure to the notice of others.

The method is fundamentally an automated colorimetric estimation of oxyhaemoglobin in solution. The sequence of events is shown in the flow diagram (Fig. 1).

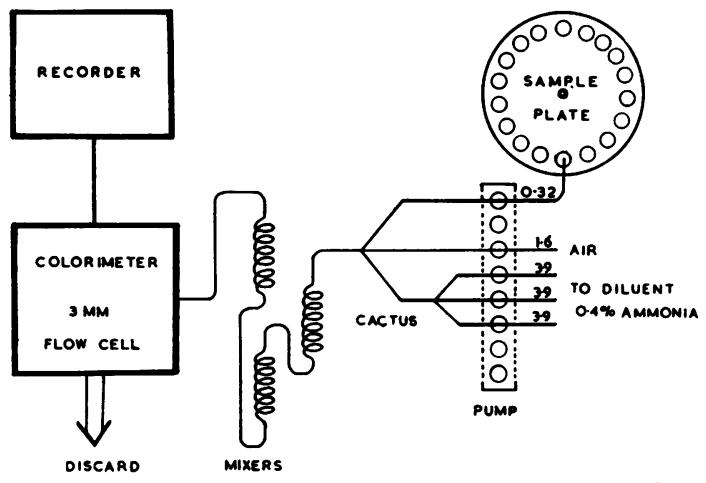

FIG. 1. Flow diagram.

Samples of blood are taken up by the sampling crook and after passing through the proportioning pump are mixed with 37 volumes of diluent. Complete mixing is achieved by a suitable arrangement of helical mixing coils. The diluted oxyhaemoglobin solution passes through a flow cell colorimeter and the imbalance in the latter is fed to an automatic graphical recorder.

\section{REAGENTS}

The only reagent required is $0.4 \%$ ammonia water. This concentration is used to achieve rapid haemolysis. It must be appreciated that all methods using the Autoanalyser

Received for publication 20 April 1961.

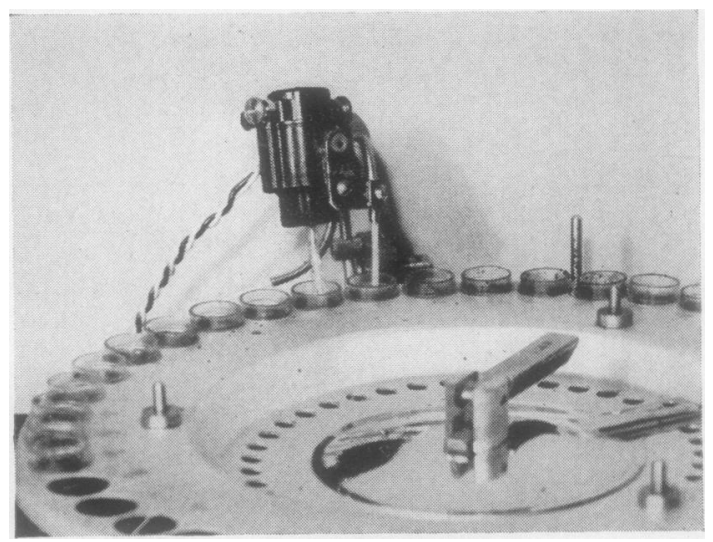

FIG. 2. The sampler plate, crook, and mixer.

require large volumes of reagents. Consequently, the diluent must be prepared in 10 litre amounts.

\section{SAMPLING}

The samples of mixed venous blood collected in $5 \mathrm{ml}$. bijou bottles containing $10 \mathrm{mg}$. EDTA are mixed in a Matburn mixer. Approximately $1 \mathrm{ml}$. aliquots from each individual sample are poured into a clean perspex sample cup which is then placed on the sampler plate.

One difficulty which presented itself was the problem of the samples of blood settling in the cups before they were presented to the sampling crook. The method recommended by the American manufacturers of bubbling air through the samples led to streaming of bubbles without the blood being completely mixed. After consultation with the London agents they adapted the sampler crook to take a small motor with a Teflon paddle. This mixed the blood sample before it was presented to the sampling crook and proved both simple and satisfactory (Fig. 2).

The possibility that the motor paddle might carry over blood from one sample to the next was found not to occur since the rapid spinning of the non-wettable Teflon paddle made it self-cleansing.

\section{THE MANIFOLD}

The various components of the tube manifold and their sizes are shown in the flow diagram (Fig. 1). It will be seen that no dialyser or heating module are required. Instead three 'tumbler' coils are included in the circuit to ensure complete haemolysis. It was found that this arrangement of the manifold made it possible to run the sampler module at 40 tests per hour without alternating samples of water with samples of blood in order to achieve complete separation in the tube lines.

\section{THE RECORDER}

The peaks obtained on the electronic recorder should have clean apices and the curve should reach zero or thereabouts between each observation. Four blood 


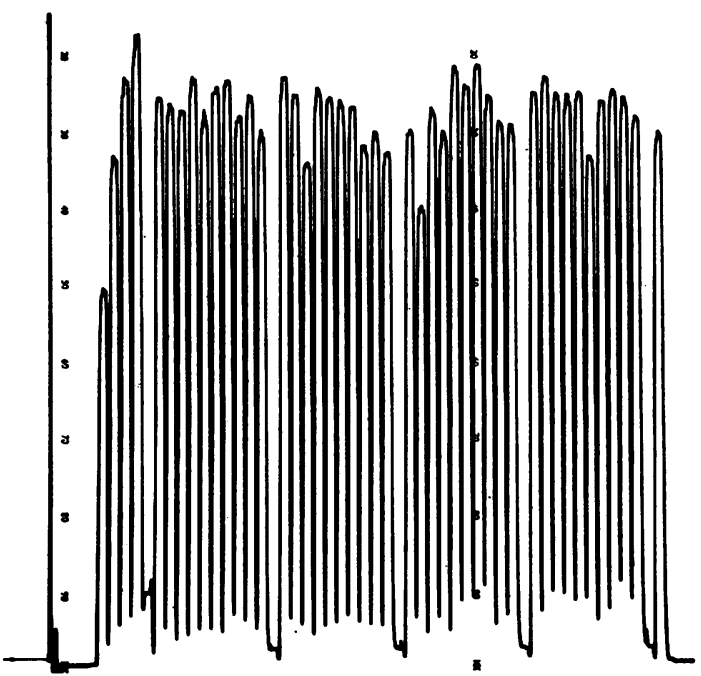

FIG. 3. Graphical record of a run of tests, including the controls.

samples of known concentration are included in each run and from these results a standard curve can be plotted. This is used to determine the concentration of haemoglobin in the unknowns. To eliminate errors in recording we have found it convenient to divide a run into blocks of 10 samples separated by a water blank which acts as a marker (Fig. 3).

\section{REPRODUCIBILITY}

Preliminary experiments demonstrated the reproducibility of the machine. A specimen of blood when subsampled and tested by the machine gave identical peaks. The same series of samples of different concentrations of haemoglobin were tested on four successive days and the results for each sample agreed to $\pm 1 \%$.

\section{ACCURACY}

The accuracy of the machine was tested by comparing the results obtained by the automatic procedure with a standard oxyhaemoglobin manual method in which the results were estimated with a standard photoelectric colorimeter. First the haemoglobin standard (Keeler) was estimated by both techniques and gave identical results. Then the haemoglobin concentrations of 150 individual samples of blood were determined in parallel by both methods. The results are plotted on a graph (Fig. 4) which shows excellent comparisons and linearity throughout the range of haemoglobin values. It can be seen that the differences between the results do not vary beyond $\pm 3 \%$ Haldane. On the whole, the automatic technique tends to give a haemoglobin value which, on average, is $0 \cdot 15 \mathrm{~g}$. lower than the manual method. This difference is not significant as the M.C.H.C.s calculated from both figures and the P.C.V. gave results in the same range.

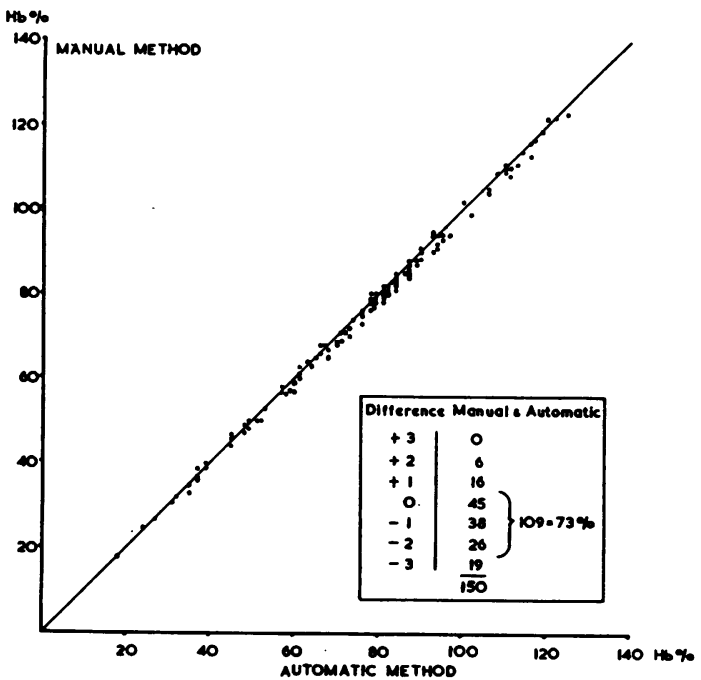

FIG. 4. Comparison of results by manual and automatic methods.

\section{DISCUSSION}

Once these performance characteristics had been ascertained the automated procedure was put into routine practice and has been in constant use for six months.

It soon became obvious that a considerable saving on technical time was being achieved. To test just how much this was, we proceeded to test 100 routine blood samples by the manual and the automatic methods. The total time required from the start to the recording of the results was much the same by both methods, namely, $1 \mathrm{hr} .54 \mathrm{~min}$. for the manual and $2 \mathrm{hr}$. $2 \mathrm{~min}$. for the automatic. However, in the manual method the technician was fully employed throughout the whole period of $1 \mathrm{hr} .54 \mathrm{~min}$., whereas in the automatic method only $37 \mathrm{~min}$. of his time was needed, namely, 30 minutes in attendance to load the sampler plate and seven minutes to read the graph and record the results. During the remaining $1 \mathrm{hr}$. $25 \mathrm{~min}$. the machine continued to take up samples and

TABLE 1

\begin{tabular}{|c|c|c|c|}
\hline \multirow[t]{2}{*}{ TECHNICIANS' TIME $F$} & \multicolumn{3}{|c|}{$\begin{array}{l}\text { FOR } 100 \text { TESTS } \\
\text { Manual }^{1}\end{array}$} \\
\hline & $h r$. & $\min$. & sec. \\
\hline $\begin{array}{l}\text { Pipetting diluent } \\
\text { Pipetting blood samples } \\
\text { Reading and recording } \\
\text { Washing pipettes } \\
\text { Total }\end{array}$ & $\begin{array}{l}\bar{z} \\
\overline{1}\end{array}$ & $\begin{array}{r}21 \\
47 \\
36 \\
8 \\
53\end{array}$ & $\begin{array}{l}25 \\
30 \\
15 \\
20 \\
30\end{array}$ \\
\hline $\begin{array}{l}\text { Loading sample plates } \\
\text { Reading and recording results } \\
\text { Total }\end{array}$ & $\begin{array}{l}\text { Aut } \\
- \\
-\end{array}$ & $\begin{array}{r}30 \\
7 \\
37\end{array}$ & $\begin{array}{l}\mathbf{0} \\
\mathbf{0} \\
\mathbf{0}\end{array}$ \\
\hline
\end{tabular}

${ }^{1}$ A verage of three technicians 
record the results automatically. As a result of this saving of technicians' time it has been possible to deviate the technicians into performing more complex procedures where automation has not been possible.

We are aware that the capital cost of installing this equipment for this single purpose may deter some management committees. For our part we are satisfied that the money expended has been justified in the accuracy of the results obtained and the saving of technical time simultaneously achieved.

The fundamental basis of any automatic device is that it must be in constant use during the working day in order to get maximum value from it. Our work load is such that we are able to keep the Autoanalyser fairly fully occupied every day doing the haemoglobin determinations alone. In smaller laboratories which cannot employ the machine whole time for this purpose, it is possible, by using the remainder of the apparatus, to carry out other chemical estimations for which the machine is specifically designed.

Another criterion of successful automation of a laboratory procedure is that the machine must be easy to operate and be free from maintenance and service difficulties. We have found during the six-month period that this automated procedure has been in daily routine use that it has been trouble-free. The only routine replacements so far required have been the ink for the graphical recorder, Teflon tubing for the manifold, and servicing of the minimotor stirrer. The latter becomes noisy after constant use and brushes require replacement. Eventually, the small motor will wear out but this replacement is simple and cheap. We have found that a new motor is required after two to three months' constant use.

The main criticism of the replacement of a manual by an automatic method in a hospital laboratory is that training of the junior technicians is impaired. This might be true of some techniques but is not so valid when levelled at haemoglobinometry, because the hand method has still to be retained for the occasional venous sample on which the result may be required in an emergency, as well as for the determination of haemoglobin in "capillary' blood. As a result, the technician has still to perform the manual method but instead of carrying out a large number of haemoglobin estimations by hand he does only a percentage. This has, in our department, worked out at somewhat less than 2,000 haemoglobins per year. This number of tests is probably as many as a technician is likely to be called upon to do in a small laboratory. There seems little point in the endless repetition of a familiar method to the point of boredom and consequent possible inaccuracy.

In the development of this automated procedure, we should like to express our thanks first to our hospital committee for a generous grant towards 'automation' in our laboratory. This is but one of many uses to which this money has been put. The London agents of the Autoanalyser have been most helpful in setting up the method and solving the mixing problem on the sampler plate. All our technicians have contributed both interest and toil to the project which they now regard as amply rewarded.

\section{Improvement of the uric acid determination by the carbonate method for serum and urine}

F. EICHHORN, S. ZELMANOWSKI, E. LEW, A. RUTENBERG, AND B. FANIAS From the Rappaport Laboratories, Biochemical Section, Beilinson Hospital, Petah Tikva, Israel

SYNOPSIS An improved colorimetric procedure for determining uric acid by the carbonate method in serum and urine is described, using a $20 \%$ sodium carbonate solution with urea. Reliable results are also obtained in high concentrations of uric acid.

The first method for determining uric acid colorimetrically was based on the reduction of phosphotungstic acid by uric acid in the presence of concentrated sodium carbonate (Folin and Denis, 1912-13). Later the carbonate was replaced by cyanide (Folin, 1930) to intensify the colour of the reaction mixture to be read visually. But the cyanide method has several drawbacks: a poisonous solution must be handled, the colour developed is not always reproducible, at concentrations of uric acid above $6 \mathrm{mg}$. \% the intensity of the developed colour is too weak and thus results are too low. When such high concentrations are encountered, the test must be repeated with smaller amounts of serum or diluted Folin-Wu filtrate. We observed the loss of sensitivity in the cyanide method at high concentrations of uric acid while experimenting with uricase. With uricase even 12 to $14 \mathrm{mg}$. \% of uric acid could be detected, while with cyanide this could not be done unless smaller amounts of serum were used.

We tried using strong alkaline coupled with weak acid, such as sodium silicate, sodium borate, and sodium glycerophosphates, as a possible replacement for cyanide. The best results, however, were not obtained with these reagents but with sodium carbonate, as in the original method (Folin and Denis, 1912-13). Carbonate was also introduced anew by Caraway (1955). In his method the phosphotungstic acid reagent is used at a dilution of $1: 10$ and ensures the final alkaline $p \mathrm{H}$ of 10 needed for satisfactory development of colour. Under these conditions, however, the sensitivity of the method is decreased. For higher concentrations of uric acid (above $5 \mathrm{mg}$. \%) this amount of reagent does not suffice and the standard curve ceases to be linear. A larger amount of the acid reagent may be introduced and kept at $p \mathrm{H} 10$ and $20 \%$ of sodium carbonate instead of $10 \%$ is used. Under these conditions the colour is intensified. However, at this concentration of carbonate a white precipitate appears and makes the photometric determination impossible.

Received for publication 1 November 1960. 